\title{
Serum albumin-protamine conjugate for biocompatible platform for targeted delivery of therapeutic macromolecules
}

\author{
Shahriar Absar, ${ }^{1}$ Kamrun Nahar, ${ }^{1}$ Suna Choi, ${ }^{1}$ Fakhrul Ahsan, ${ }^{1}$ Victor C. Yang, ${ }^{2}$ \\ Young M. Kwon ${ }^{1,3}$ \\ ${ }^{1}$ Department of Pharmaceutical Sciences, School of Pharmacy, Texas Tech University Health Sciences Center, \\ 1300 Coulter St, Amarillo, Texas 79106 \\ ${ }^{2}$ Department of Pharmaceutical Sciences, College of Pharmacy, University of Michigan, 428 Church St, \\ Ann Arbor, Michigan 48109 \\ ${ }^{3}$ Department of Pharmaceutical Sciences, College of Pharmacy, Nova Southeastern University, \\ 3200 S. University Dr., Fort Lauderdale, Florida 33328, USA
}

Received 28 June 2013; revised 31 July 2013; accepted 9 August 2013

Published online 27 August 2013 in Wiley Online Library (wileyonlinelibrary.com). DOI: 10.1002/jbm.a.34916

\begin{abstract}
A well-defined, one-to-one conjugate between human serum albumin (HSA) and protamine was synthesized and characterized as a biocompatible carrier for macromolecules. In circulation, the conjugate will camouflage drug molecules upon complex formation, while liberating free drug at the desired location using a triggering mechanism. The $\mathrm{N}$-terminus of protamine was thiolated and conjugated with the unpaired Cysteine-34 of HSA, and was purified by ionexchange chromatography. The molecular weight of the conjugate was $70.8 \mathrm{kDa}$, confirming one-to-one conjugation between HSA (66.6 KDa) and protamine (4200 Da). Superimposed fluorescence spectra of native HSA and HSA-protamine conjugate indicated no conformational change around the Trp-214. The conjugate had marked reduction in hemolytic and cytotoxic properties compared to protamine. When
\end{abstract}

therapeutic potential was tested using tissue plasminogen activator as a model drug, HSA-protamine conjugate suppressed the enzymatic activity by $65 \%$, which was fully recovered by a triggering agent, heparin. The construct showed binding characteristics with activated platelets upon conjugation with a targeting peptide, demonstrating flexibility to introduce suitable homing moiety on the surface. The camouflaged construct retained triggered release property in human plasma condition. Overall, the conjugate has a good potential to serve as a biocompatible platform for macromolecular drugs. (c) 2013 Wiley Periodicals, Inc. J Biomed Mater Res Part A: 102A: 2481-2490, 2014.

Key Words: albumin-based delivery, localized thrombolysis, prodrug strategy for macromolecules, albumin as a carrier

How to cite this article: Absar S, Nahar K, Choi S, Ahsan F, Yang VC, Kwon YM. 2014. Serum albumin-protamine conjugate for biocompatible platform for targeted delivery of therapeutic macromolecules. J Biomed Mater Res Part A 2014:102A:2481-2490.

\section{INTRODUCTION}

The use of human serum albumin (HSA) as a carrier for therapeutic molecules has been under extensive investigation. HSA itself serves as a therapeutic agent in the events of severe burns, hypovolemia, surgical blood loss, acute or chronic liver failure, cardiopulmonary bypass, nutrition support, and so forth. ${ }^{1}$ Furthermore, the ability of HSA to bind with multiple ligands, and consequently serve as a depot for numerous exogenous and endogenous substances translated the use of HSA as a drug carrier from bench to bedside. An example includes development of insulin Levemir ${ }^{\circledR}$ which contains myristic acid at the $\epsilon$-amino group that binds with HSA after administration into the body, resulting in a 50 - to 70 -fold increase in the half-life of the drug. $^{2}$ The albumin-paclitaxel nanoparticle, Abraxane ${ }^{\circledR}$, becomes soluble upon intravenous administration, mimicking endogenous albumin. These nanoparticles are transported into the interstitium of a solid tumor through the interaction of albumin with the glycoprotein gp60 that is expressed on the endothelial cell surface ${ }^{3-5}$ Recently, cell penetrating albumin conjugates have also been reported for intracellular delivery of doxorubicin. ${ }^{6}$ Furthermore, Kratz and co-workers proposed an albumin-based pro-drug approach by conjugating doxorubicin to cysteine34 of endogenous HSA. ${ }^{7,8}$ The prodrug reduced the systemic toxicity with a favorable cytotoxic profile when the active drug was released from albumin, either in the mild acidic environment of the tumor extracellular compartment or intracellularly in the acidic lysosomal compartment. ${ }^{9}$ Compared to the promising outcome of this approach with small molecules, there are rooms for advancement in the development of albumin-based drug carrier for macromolecules, such as enzyme drugs that are administered directly into the vascular compartment. 
Therapeutic proteins, enzymes, oligoneucleotides, and monoclonal antibodies have gained significant attention because of their highly specific action on the respective substrates. However, in many cases, the molecular targets are present throughout the circulation causing the macromolecules unable to distinguish the targets in the diseased site from those in general tissues generating off-target effects. An effective way to overcome this shortcoming could be targeted delivery, which, however, still carries the potential for acting on off-target substrates while traveling in the circulation. Thus, a pro-drug approach for these biomacromolecules is warranted to suppress the activity of the drugs in the systemic circulation, and regenerate at the target site. Being the most abundant plasma protein, HSA can serve as a camouflage for therapeutic agents in the systemic circulation. ${ }^{10,11}$ To that end, we propose an albumin-based prodrug construct for enzyme drugs in which the drug will be carried by albumin via electrostatic interaction that will be disrupted by a triggering agent upon reaching the target. As a model macromolecule, we have used tissue plasminogen activator (tPA) that produces enzymatic action by converting plasminogen to plasmin, which further degrades fibrin mesh to restore blood flow to an occluded vessel. However, tPA possesses lifethreatening bleeding risk because of its indiscriminate action on plasminogen. ${ }^{12}$ Therefore, a prodrug approach to minimize ubiquitous plasmin generation while maximizing tPA's exposure at the clot site could significantly attenuate bleeding complication and enhance therapeutic outcome. Whereas the accumulation of the agent at the clot site can be achieved by using a suitable homing moiety, regeneration of activity remains a critical challenge because of lack of triggering environment, that is, $\mathrm{pH}$, temperature, or reducing agent, during a thrombotic event. As heparin is commonly used in the therapeutic management of thrombotic events, we have designed the construct such that the regeneration of activity can be achieved by heparin administration. Hence, a one-to-one conjugate between albumin and protamine was developed (Fig. 1). On the other hand, tPA, the most common thrombolytic agent, was modified to introduce a series of negative charges so that it forms an electrostatic complex with the protamine moiety of the albumin-protamine conjugate. We chose the homing moiety based on the interaction of fibrinogen with integrin GPIIb/IIIa that is expressed on activated platelets, the underlying factor of aggregation and formation of occlusive thrombus. Ware et al. reported the C-terminal gamma chain peptide sequence of fibrinogen, CQQHHLGGAKQAGDV, as the central structural segment responsible for the binding, ${ }^{13}$ and hence was used as targeting moiety. Thus, the construct will accumulate on the thrombus following which therapeutically active tPA will be released upon interaction with subsequently administered heparin. In the current work, we have synthesized and purified albumin-protamine conjugate, characterized for vascular and hemo-compatibility and demonstrated the feasibility to be used for a targeted prodrug construct for thrombolytic enzyme.

\section{MATERIALS AND METHODS Materials}

HSA (from human blood source), protamine, fibrinogen, plasminogen, aldrithiol (2,2'-Dithiopyridine 2,2' -Dipyridyldi-

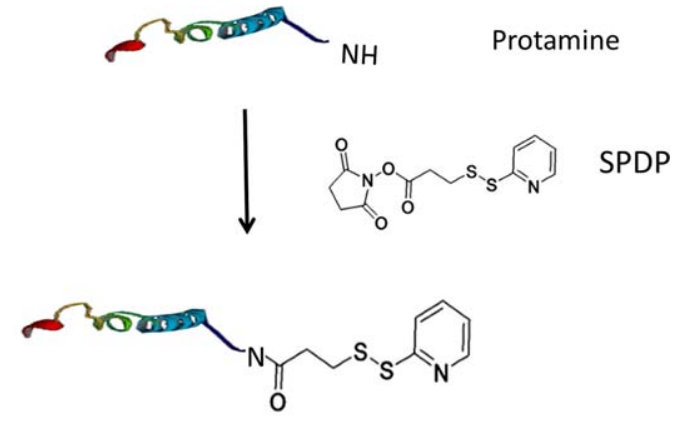

Pyridyldithiol-activated protamine

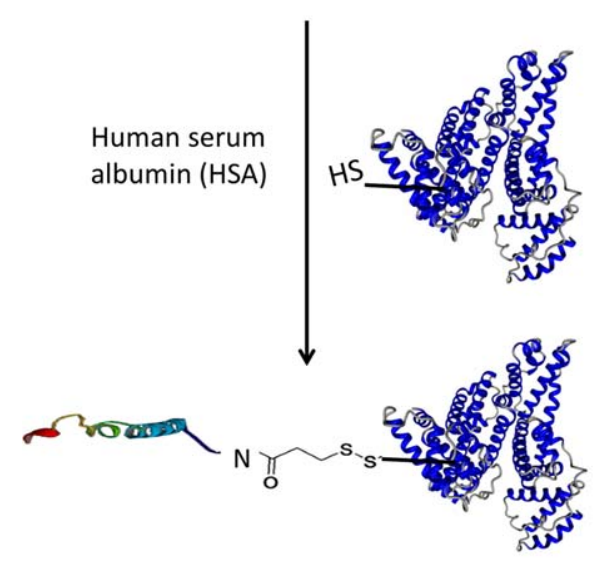

HSA-protamine conjugate

FIGURE 1. Graphical representation of one-to-one conjugate preparation via Cys-34 of HSA and N-terminus (secondary amine) of protamine. The three-dimensional structure of HSA was generated by PPG: the Protein Picture Generator http://bioserv.rpbs.jussieu.fr/PPG; DINO: Visualizing Structural Biology (2002) http://www.dino3d.org. ${ }^{21}$ Atomic coordinates were downloaded from the Protein Data Bank, accession number 4iw2. [Color figure can be viewed in the online issue, which is available at wileyonlinelibrary.com.]

sulfide), dithiothreitol (DTT) were purchased from SigmaAldrich (St. Louis, MO). N-Succinimidyl1,3-(2-pyridyldithio) propionate (SPDP) was obtained from Molecular Biosciences (Boulder, CO). tPA was purchased from Genentech (Genentech, South San Francisco, CA). LMWH was obtained from Eisai (Woodcliff Lake, NJ). The plasmin specific chromogenic substrate S-2251 was obtained from Chromogenix (Lexington, MA). Fluorescein isothiocyanate (FITC) was purchased from Fisher Scientific (Pittsburgh, PA). The 7-mers of glutamate oligopeptide (CEEEEEEE) and the targeting peptide sequence 14-mers of the C-terminal peptide (CQQHHLGGAKQAGDV) from the C-terminus of fibrinogen gamma chain were obtained from EZBiolab (Carmel, IN). All the cell culture related materials were obtained from American Type Cell Collection (ATCC; Manassas, VA). All other buffers and chemical reagents were purchased from Sigma-Aldrich and of reagent grade.

\section{Activation of $\mathbf{N}$-terminus proline of protamine}

Protamine (10 mg) was taken in a scintillation vial into which $770 \mu \mathrm{L}$ of $2 M \mathrm{NaCl}$ dissolved in $20 \mathrm{mM}$ phosphate 
buffer was added. The mixture was gently stirred at room temperature for $10 \mathrm{~min}$. Separately, $120 \mathrm{mg}$ of polyethylene glycol-600 was dissolved in $770 \mu \mathrm{L}$ of $100 \mathrm{mM}$ phosphate buffer and added to protamine solution. Once protamine is completely dissolved, SPDP, dissolved in $550 \mu \mathrm{L}$ dimethylformamide (DMF), was added drop-wise at a feed-ratio of 5, 10,15 , and 20 -fold excess of protamine. The reaction was continued for $4 \mathrm{~h}$ at room temperature, followed by quenching with $100 \mu \mathrm{L}$ of $100 \mathrm{~m} M$ glycine. Excess SPDP was purified by Fast Protein Liquid Chromatography (FPLC, Biologic Duo Flow, Biorad) using ion-exchange CM-Sepharose column (GE Healthcare) with elution buffer (A) $20 \mathrm{mM}$ phosphate buffer and (B) $2 \mathrm{mM} \mathrm{NaCl}+20 \mathrm{mM}$ phosphate at $\mathrm{pH}$ 7.4. To quantify the yield of activated protamine, $900 \mu \mathrm{L}$ of the collected sample was taken in a quartz cuvette and absorbance was measured at $343 \mathrm{~nm}$ by Diode array spectrophotometer (HP 8453A, Olis). Then, $100 \mu \mathrm{L}$ of $0.5 M$ DTT solution was added and, following $15 \mathrm{~min}$ of incubation, the absorbance at 343 was again measured. The release of pyridyl-2-thione upon treatment of DTT was calculated to determine the extent of protamine activation.

Synthesis and purification of HSA-protamine conjugate HSA (10 mg) was dissolved in $20 \mathrm{mM}$ phosphate buffer at pH 7.2 from which $500 \mu \mathrm{L}$ was taken and added drop wise to $500 \mu \mathrm{L}$ of activated protamine (protamine-PDP) solution. The reaction kinetic was monitored by Diode array spectrophotometer at $343 \mathrm{~nm}$ by measuring the release of pyridine-2-thione with time. Once the reaction progression was confirmed, the feed-ratio was optimized by introducing $1,2,3$, and 4-fold molar excess of HSA to the activated protamine and incubating at room temperature for $2 \mathrm{~h}$ with mild shaking. The HSA-protamine conjugate was then purified in one-step using ion-exchange CM-Sepharose column with the same elution buffer A and B as mentioned in Activation of $\mathrm{N}$-terminus proline of protamine" section. The yield of the conjugate was determined by UV spectroscopy at $280 \mathrm{~nm}$.

\section{Determination of molecular weight and fluorescence spectra of HSA-protamine conjugate}

The molecular weight of the prepared HSA-protamine conjugate was determined by electrospray mass spectroscopy (Agilent 6520 Q-TOF). An aliquot of $40 \mu \mathrm{L}$ sample at a concentration of $1 \mathrm{mg} / \mathrm{mL}$ was loaded into the autosampler vial from which $5 \mu \mathrm{L}$ was injected into a $\mathrm{C}-8$ poroshell column. Buffer A, composed of $5 \%$ acetonitrile with $0.1 \%$ formic acid / 95\% water with $0.1 \%$ formic acid, was run through the sample loaded column for $2 \mathrm{~min}$ at a flow rate of $0.5 \mathrm{~mL} / \mathrm{min}$. Then a gradient to $100 \%$ acetonitrile with $0.1 \%$ formic acid was run over $10 \mathrm{~min}$. The mass spectrometer was run in positive ion mode, with reference mass correction on a dual ESI source. The data were processed with Agilent's MassHunter software. To compare the prepared HSA-protmaine conjugate with native HSA (1 mg/mL), a similar run was performed keeping all the parameters same as that of the sample. For fluorescence spectroscopic study, a sample $(1 \mathrm{mg} / \mathrm{mL})$ of HSA and HSA-protamine conjugate was taken in a cuvette with 1-cm path length and measurement was performed on Hitachi F-2500 fluorescence spectrophotometer. The spectrum was recorded in the range of 300-400 nm using excitation wavelength of $295 \mathrm{~nm}$.

\section{Hemolysis of protamine-conjugated HSA carrier}

Hemolysis of the prepared HSA-protamine was monitored, which could be originated from the cationic protamine moiety. Human blood sample was collected from Coffee Memorial Blood Center (Amarillo, TX). An aliquot of $100 \mu \mathrm{L}$ of blood cells were taken and diluted to $1 \mathrm{~mL}$ with saline. The cells were washed three times using centrifugation (Legend Micro 17R, Thermo Scientific) and the final volume was made $1 \mathrm{~mL}$. The cells were further diluted 10 times from which $100 \mu \mathrm{L}$ was taken in each eppendorf tube into which protamine or HSA-protamine conjugate was added at a final protamine concentration of $10 \mu \mathrm{g} / \mathrm{mL}$. The final volume of $1 \mathrm{~mL}$ was incubated at $37^{\circ} \mathrm{C}$ for different time points. Deionized water and saline was used as positive and negative controls, respectively. Following incubation, the samples were centrifuged at $800 \mathrm{~g}$ for $2 \mathrm{~min}$ and the supernatants were analyzed for hemolysis by measuring absorbances at $540 \mathrm{~nm}$ on a spectrophotometer.

\section{Cytotoxicity study of prepared carrier system}

The cytotoxicity of HSA-protamine conjugate was tested with arterial (pulmonary) smooth muscle cells using MTT assay. ${ }^{14,15}$ Cells were cultured in Dulbecco's Modified Eagle's Medium containing Fetal Bovine Serum and penicillin/streptomycin. 50,000 cells were seeded on 96-well flat bottom plate and kept overnight for settling down. On the following day, protamine or HSA-protamine conjugate was separately added to the wells at a concentration range from 0 to 1 $\mathrm{mg} / \mathrm{mL}$ equivalent of protamine, and incubated for $4 \mathrm{~h}$ at $37^{\circ} \mathrm{C}$. The culture media was then replaced by $100 \mu \mathrm{L}$ of fresh media containing $0.5 \mathrm{mg} / \mathrm{mL}$ MTT solution, and incubated for another $4 \mathrm{~h}$. After gently removing the MTT solution, the crystalline precipitate was dissolved in $100 \mu \mathrm{L}$ of dimethylsulfoxide (DMSO; molecular biology grade) and absorbance was measured at $570 \mathrm{~nm}$. The percent of viable cells were calculated by the following equation:

$$
\% \text { of Viable cells }=\left(A_{\text {treated }}-A_{\mathrm{MTT}}\right) /\left(A_{\text {control }}-A_{\mathrm{MTT}}\right) \times 100
$$

\section{HSA-protamine conjugate in the triggered-release} approach for thrombolytic macromolecules

The proof-of-principle triggered-release system for macromolecules with our prepared HSA-protamine conjugate was tested using tPA as a model drug. To introduce electronegative functionality, tPA was conjugated with low molecular weight heparin (LMWH) by previously described method. ${ }^{10}$ HSA-protamine conjugate at 50-fold excess of tPA was incubated with tPA for $30 \mathrm{~min}$ at room temperature to facilitate electrostatic interaction between LMWH and protamine moieties. The amidolytic activity of tPA upon complex formation with HSA-protamine was measured by Chromogenic assay. ${ }^{16}$ Briefly, $1.3 \mu M$ of substrate S-2251, $0.24 \mu M$ 
plasminogen and $0.5 \mu \mathrm{g} / \mathrm{mL}$ tPA dissolved in $50 \mathrm{mM}$ Tris $\mathrm{HCl}$ containing $50 \mathrm{mM} \mathrm{NaCl}$ with $0.01 \%$ Tween 80 at $\mathrm{pH} 7.2$ was taken in a $300 \mu \mathrm{L}$ well of 96 -well plate. The initial rate of hydrolysis of S-2251 by plasmin generated from plasminogen by tPA was determined by measuring the absorbance at $405 \mathrm{~nm}$ at different time intervals using a micro-plate reader (SynergyMx, Biotek). The slope of absorbance against square of time $\left(\Delta A \mathrm{~min}^{-2}\right)$ was expressed as the initial rate of S-2251 hydrolysis. To assess heparin-mediated triggered release, the assay was also performed in the presence of 0.4 $\mu / \mathrm{mL}$ of heparin.

\section{Binding study of homing-peptide decorated HSA-protamine conjugate with activated platelets} The homing peptide, CQQHHLGGAKQAGDV, was conjugated to the primary amines of HSA of the conjugate. Briefly, SPDP, 20-fold excess of HSA, was dissolved in DMF and added drop-wise to the HSA-protamine conjugate. Following 30 min incubation at room temperature, excess SPDP was removed by size-exclusion chromatography, and the resulting purified thiol-reactive conjugate was incubated with the homing peptide for $30 \mathrm{~min}$ at room temperature. Finally, the homing-peptide decorated HSA-protamine was again purified by size-exclusion chromatography. The binding characteristic of the conjugate with activated-platelets were tested by fluorescence microscopy. ${ }^{17,18}$ Human platelets $\left(1.68 \times 10^{9}\right.$ platelets $/ \mathrm{mL}$ of plasma $)$ were collected from Coffee Memorial blood center (Amarillo, TX) and were isolated by centrifuging $1 \mathrm{~mL}$ of platelet-rich plasma at $400 \mathrm{~g}$ for $15 \mathrm{~min}$ at $25^{\circ} \mathrm{C}$. After removing the supernatant, platelets were re-suspended in PBS containing 1\% Bovine Serum Albumin (BSA). The sample was diluted to make a suspension containing 250,000 platelets per $\mu \mathrm{L}$, which was then adsorbed onto collagen-coated glass cover-slips at room temperature for $1 \mathrm{~h}$ and washed thrice with PBS. To confirm the adhesion of platelet monolayer on cover-slips, cells were stained with FITC-tagged anti-GPIIb/IIIa monoclonal antibody (eBioscience, CA, USA), and subsequently observed under fluorescence microscopy (with magnification of $10 \times$ $60 \times 1.5$ (overall $900 \times$ ). FITC-labeled peptide-linked camouflaged tPA construct was incubated with the adsorbed platelets for $1 \mathrm{~h}$ in dark at room temperature. Fluorescent construct without the homing peptide was also incubated as a control. All incubations were carried out in the presence of $5 \mathrm{mM} \mathrm{CaCl}{ }_{2}$ solution under mild shaking to facilitate activation of platelet.

\section{Stability of the construct in human plasma and blood environment}

Fresh human plasma and blood samples were collected from coffee memorial blood center (Amarillo, TX). To test the stability of the electrostatic interaction between anionmodified tPA and HSA-Protamine conjugate, albumincamouflaged tPA was incubated with plasma for $30 \mathrm{~min}$ at $37^{\circ} \mathrm{C}$. An aliquot of $50 \mu \mathrm{L}$ of the incubated plasma sample was then taken and added to a well (96-well plate) to perform Chromogenic assay as described previously (section HSA-protamine conjugate in the triggered-release approach for thrombolytic macromolecules). To evaluate the triggered release profile, a heparin $(0.4 \mu / \mathrm{mL})$ treated group was also tested with the camouflaged tPA group.

\section{Statistical analysis}

All data were presented as the mean \pm SD. One-way ANOVA was performed to compare among the groups and analyze the statistical significance. Post-hoc analyses were conducted using Tukey's comparison (GraphPad Prism, version 5.0, GraphPad Software, San Diego, CA). $p$-values less than 0.05 were considered statistically significant.

\section{RESULTS}

\section{$\mathrm{N}$-terminal thiolation of protamine}

As protamine does not contain any lysyl residues, the only site to introduce the SPDP linker at neutral to slightly alkaline condition is the $\mathrm{N}$-terminal proline, a secondary amine. ${ }^{19}$ However, addition of SPDP in either ethanol or DMF caused precipitation of protamine when the reaction media was phosphate buffered saline, which was later solved performing the reaction in $2 M \mathrm{NaCl}$ stabilized with PEG-600. FPLC chromatographic data demonstrate that the purification method effectively removed unreacted SPDP [Fig. 2(A)]. Protamine activation was qualitatively confirmed by the release of pyridine-2-thione at $343 \mathrm{~nm}$ upon treatment with DTT [Fig. 2(B)]. Quantification indicates that as much as $41 \%$ of protamine was activated by SPDP when 5 -fold excess of SPDP was used. The extent of activation increased to $63 \%$ when amount of SPDP was increased by 2-fold. However, further increase in SPDP mole\% did not produce any increase in protamine activation [Fig. 2(C)]. Hence, a 10-fold excess of SPDP over protamine was selected as the optimum feed ratio.

\section{Preparation of HSA-protamine conjugate}

Addition of HSA solution into activated protamine spontaneously released pyridine-2-thione [Fig. 3(A)], indicating the reaction between the Cys-34 of HSA and activated protamine. When protamine solution, without SPDP modification, was reacted with HSA, no reaction was evident by spectroscopy. The kinetics of the reaction revealed that it reaches a plateau at around 10 min [Fig. 3(B)]. The one-step purification method effectively separated unreacted HSA, unreacted activated protamine, and the HSA-protamine conjugate. Because of no affinity towards CM-sepharose column, unreacted HSA elutes immediately after being injected, which was confirmed by separate injection (data not shown). The reason that HSA-protamine conjugate elutes separately from unreacted activated protamine may be attributed to the slight decrease in binding affinity of the bulky HSA-protamine to CM-Sepharose resin. With the increasing feed-ratio of HSA over protamine-PDP, an increase in the peak for HSA-protamine conjugate was observed with corresponding decrease in the protaminePDP peak, suggesting the depletion of protamine with increasing production of the conjugate [Fig. 3(C)]. A 4:1 molar ratio of HSA:protamine resulted with almost complete depletion of protamine. Quantification of HAS-protamine 


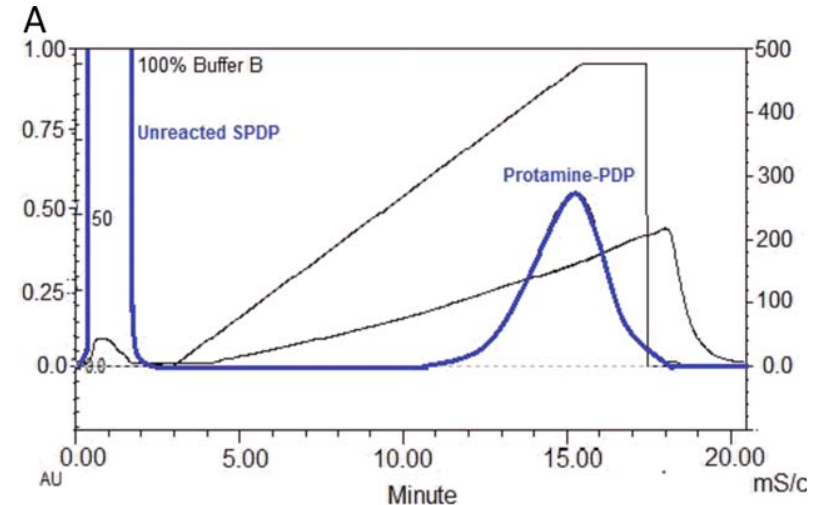

B
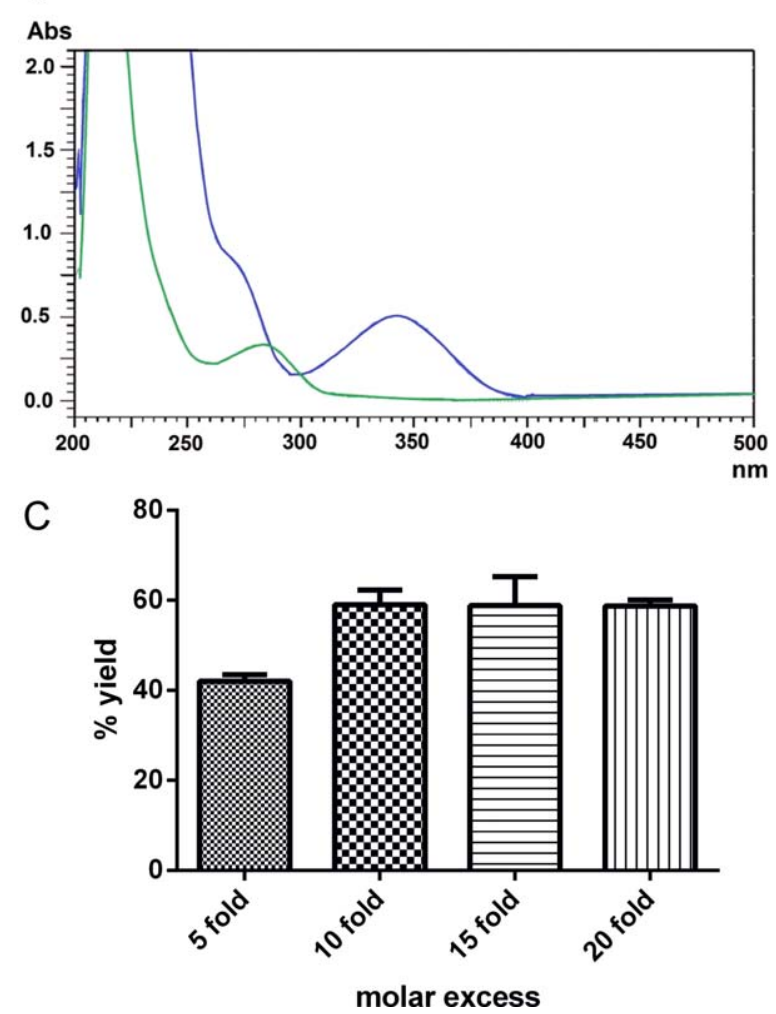

FIGURE 2. (A) Purification of PDP-activated protamine by ionexchange chromatography using $\mathrm{CM}$-sepharose column. The $1 \mathrm{~mL}$ reaction mixture was eluted using buffer $\mathrm{A}$ : $20 \mathrm{mM}$ phosphate, $\mathrm{pH}$ 7.4; B: $20 \mathrm{mM}$ phophate $\mathrm{pH} 7.4+2 \mathrm{M} \mathrm{NaCl}$. (B) Release of pyridyl-2thione measured at $343 \mathrm{~nm}$ upon treatment of PDP-activated protamine with DTT. The green and blue lines represent chromatographic traces of PDP-activated protamine before and after addition of DTT, respectively. (C) \% Yield of PDP-activated protamine with increasing mol:mol feed ratio of SPDP to protamine. [Color figure can be viewed in the online issue, which is available at wileyonlinelibrary.com.]

conjugate also demonstrated that the yield of the conjugate increased with increasing HSA molar ratio from 1:1 to $4: 1$ as that of protamine [Fig. 3(D)]. Thus, a 4 -fold excess of HSA was selected for further preparation of the conjugate.

\section{Characterization of HSA-protamine conjugate}

The goal of this conjugation reaction is the formation of one-to-one conjugate between HSA and protamine to avoid heterogeneity in drug delivery application. The electrospray mass spectroscopy data revealed that the molecular weight of the conjugate was around $70.8 \mathrm{KDa}$ [Fig. 4(B)]. As the molecular weight of native HSA was found to be $66.6 \mathrm{kDa}$ [Fig. 4(A)] and that of protamine was $4.2 \mathrm{kDa}$, the weight of the conjugate demonstrates a 1:1 conjugation. Furthermore, the intrinsic fluorescence spectrum of HSA-protamine was analyzed to determine if protamine conjugation had altered the conformation of HSA around the tryptophan residue (Trp 274). Upon excitation at $295 \mathrm{~nm}$, both native HSA and the HSA-protamine conjugate demonstrated maximum emission intensity at $340 \mathrm{~nm}$. Moreover, the emission profile was nearly identical and resulted in superimposed spectra between native HSA and HSA-protamine conjugate [Fig. $4(C)$ ], indicating no quenching of the intrinsic fluorescence of Trp-214 residue of HSA. This indicates that conformation of the hydrophobic binding pocket in the second $\alpha$-helix domain of HSA remained unaffected after conjugation with protamine. ${ }^{20}$

\section{Biocompatibility of HSA-protamine conjugate}

The potential hemolysis, if occurs because of a high positive charge density of protamine, can limit the application of the proposed conjugate as a drug delivery tool. The hemolysis study demonstrated that protamine, in the form of HSA-protamine conjugate, had produced no lytic effect and was comparable to that of saline at equivalent protamine concentration of $10 \mu \mathrm{g} / \mathrm{mL}$, whereas native protamine, at the same concentration, caused considerable hemolysis [Fig. 5(A)]. As the maximum concentration of protamine in human blood with our proposed delivery system would be $\leq 4 \mu \mathrm{g} / \mathrm{mL}$, we claim the conjugate hemocompatible. Further, the compatibility study in the vasculature revealed that the cell viability was almost unaffected with HSA-protamine conjugate as demonstrated by $>90 \%$ survival at a concentration equivalent to $1 \mathrm{mg} / \mathrm{mL}$ of protamine. However, cell viability was significantly compromised (only 20\% survival) at similar concentration of native protamine [Fig. 5(B)]. Thus both the hemocompatibility and cellular toxicity studies indicate that the proposed conjugate is unlikely to exert any deleterious effect at a concentration that will be applied for its use as a therapeutic cargo.

\section{Proof-of-principle application of HSA-protamine} conjugate as a carrier for therapeutic macromolecule The sole purpose of this conjugate is to mask the enzymatic activity of a macromolecule, tPA in this case, by HSA, and to regenerate the activity using therapeutic steady-state concentration of heparin. In order to form the electrostatic complex between tPA and HSA, we have previously functionalized tPA with electronegative charge. ${ }^{10}$ When the HSA-protamine conjugate was introduced to tPA with electronegative functionality, $\sim 65 \%$ of tPA's activity was suppressed (Fig. 6). However, when heparin $(0.4 \mu / \mathrm{mL})$ was added to the "camouflaged" tPA construct, the activity was fully regenerated, suggesting that while HSA masked tPA's activity by forming electrostatic complex with 

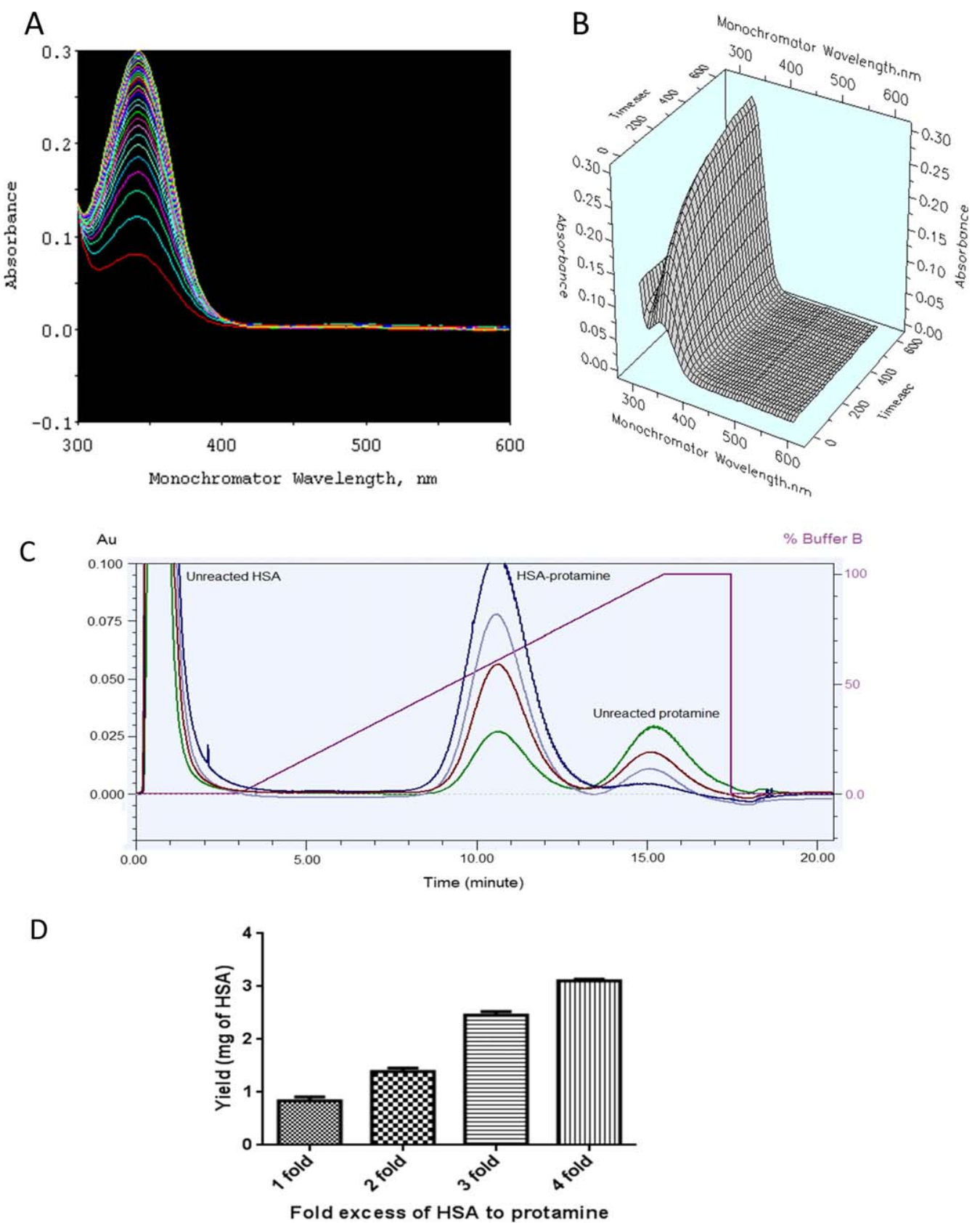

FIGURE 3. (A) Spontaneous reaction between HSA and PDP-activated protamine as monitored by the release of pyridyl-2-thione at 343 nm. The absorbance at 343 increased with increasing time. (B) Time course of HSA-protamine-PDP reaction: a 3D spectroscopic trace of the reaction from diode-array spectrophotometer demonstrating a saturation point at around $10 \mathrm{~min}$. (C) One-step purification of HSA-protamine conjugate from unreacted HSA and protamine-PDP using ion-exchange chromatography. The 1-mL reaction mixture was eluted on a CM-sepharose column with elution buffer $\mathrm{A}$ : $20 \mathrm{mM}$ phosphate, $\mathrm{pH} 7.4$; $\mathrm{B}: 20 \mathrm{mM}$ phosphate $\mathrm{pH} 7.4+2 \mathrm{M} \mathrm{NaCl}$. The green, red, violet, and blue lines represent 1, 2, 3, and 4-fold molar excess of HSA to protamine, respectively. (D) Yield of HSA-protamine conjugate with increasing molar excess of HSA to that of protamine. [Color figure can be viewed in the online issue, which is available at wileyonlinelibrary.com.]

electronegative tPA surface, administration of heparin disrupted the construct and liberated free tPA because of its strong affinity towards protamine. This observation indicates that if the camouflaged structure can be directed towards thrombus using a homing mechanism, the systemic "lytic" effect of tPA can be attenuated and the thrombolytic action can potentially be regenerated by heparin, which is commonly administered during thrombosis management.

\section{Binding of homing peptide-decorated HSA-protamine} conjugate with activated platelets

The proposed targeted/triggered delivery system using HSA-protamine conjugate is contingent upon the accumulation of the macromolecule on its site of action, which in this case is thrombus. Hence, the surface of HSA-protamine conjugate was decorated with the homing peptide that binds with GPIIb/IIIa present on activated platelets during 
A

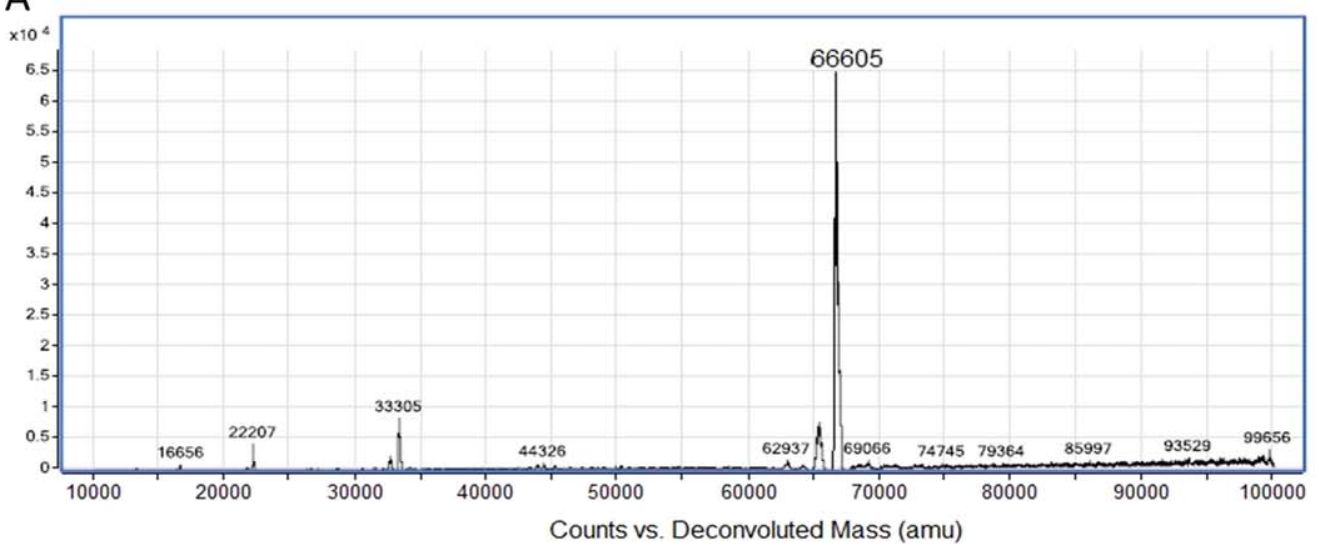

B

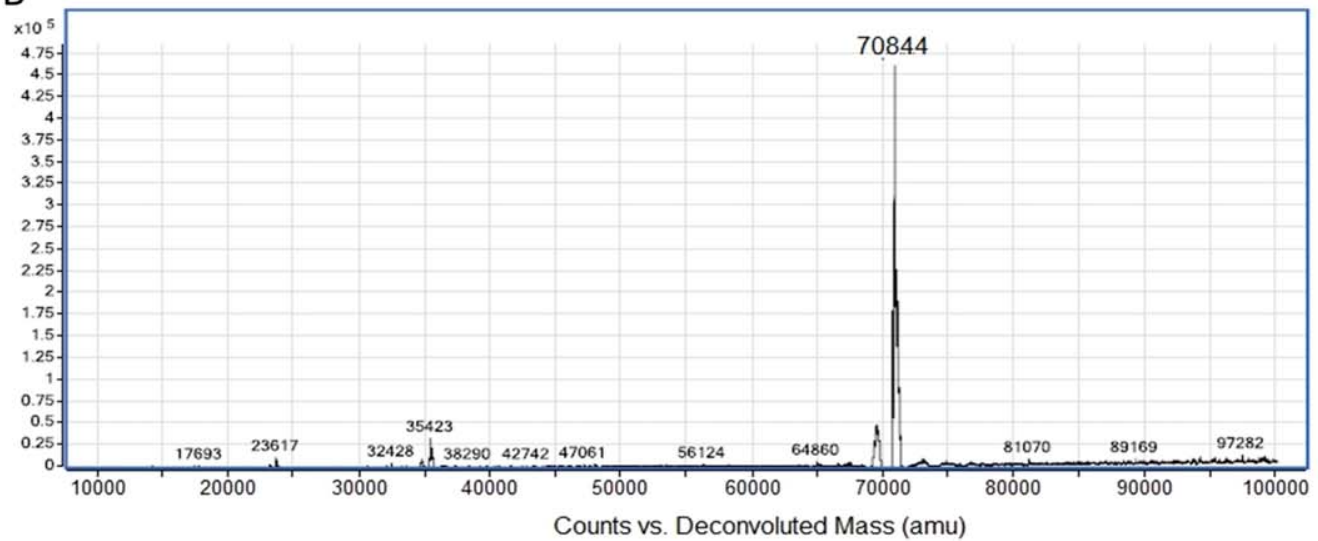

C

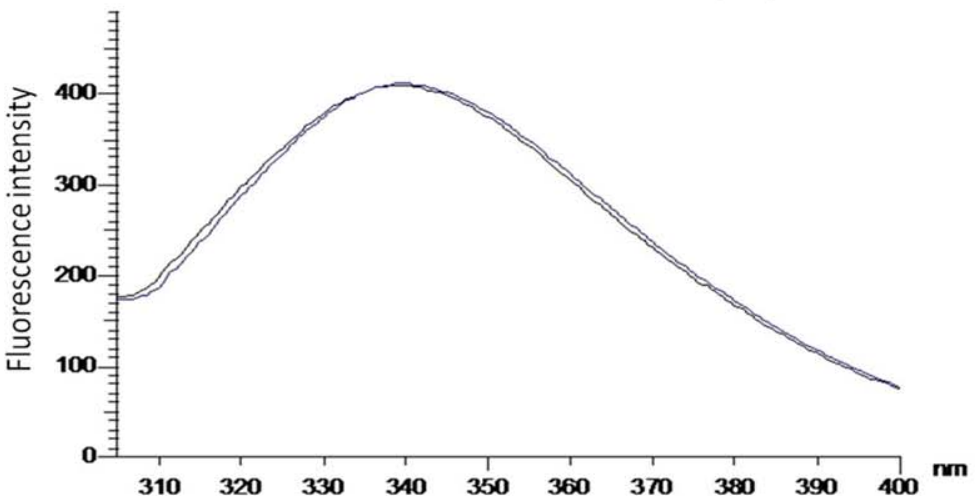

FIGURE 4. Electrospray mass spectroscopy of (A) native HSA and (B) HSA-protamine conjugate, indicating molecular weight of each species. (C) Overlapping intrinsic fluorescence emission spectra of HSA and HSA-protamine conjugate. [Color figure can be viewed in the online issue, which is available at wileyonlinelibrary.com.]

thrombotic event. Fluorescence microscopic image revealed that FITC-labeled peptide-grafted conjugate bound to the activated platelet surface as demonstrated by the staining of almost all the cells (Fig. 7). However, fluorescent conjugate without the homing peptide did not exhibit any binding with platelets. In the control experiment, activation of platelets was confirmed by the staining of the cells with FITClabeled anti-GPIIb/IIIa antibody. Thus the binding study suggests that HSA-protamine conjugate can be decorated with a suitable homing peptide to direct the cargo at a desired site.

\section{Stability of the HSA-mediated construct in human plasma}

The electrostatic interaction between HSA-protamine conjugate and anion-functionalized tPA was stable enough to mask the enzymatic activity of tPA in human plasma (Fig. 8). Moreover, the characteristic regeneration of activity was retained upon the treatment with heparin. The activity of the camouflaged tPA after heparin addition was $\sim 3$-fold higher than that of plain tPA. As heparin stimulates tPA activity both in vitro and in vivo, ${ }^{21}$ results were compared with the control group that was also treated with heparin. 
A

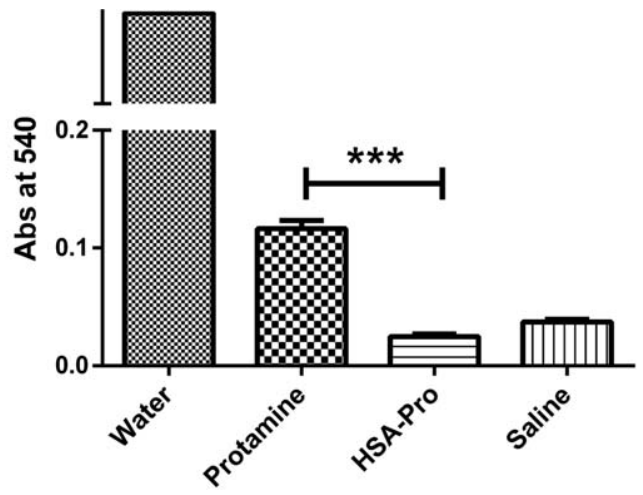

B

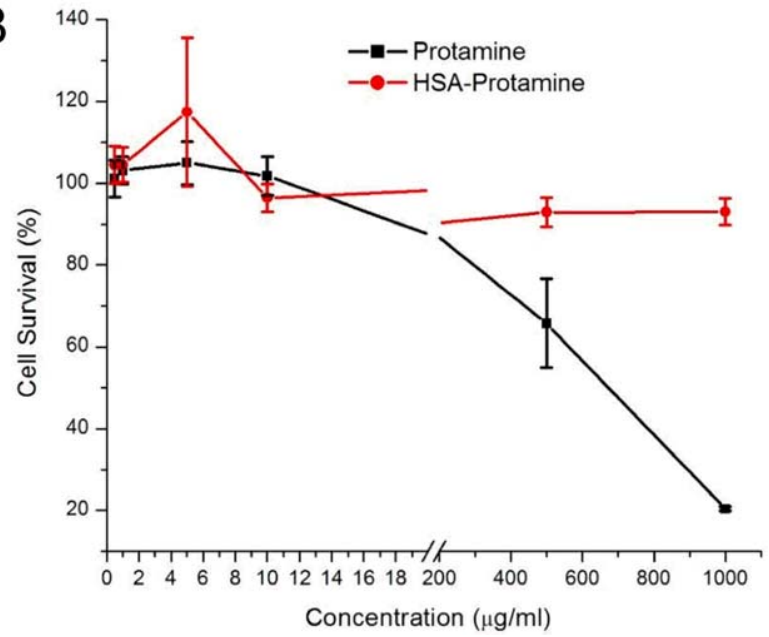

FIGURE 5. (A) Hemo-compatibility of HSA-protamine conjugate. The extent of hemolysis was determined by measuring the absorbance of released hemoglobin at $540 \mathrm{~nm}$. An equivalent protamine concentration of $10 \mu \mathrm{g} / \mathrm{mL}$ was used for both protamine and HSA-protamine groups $(n=3)$. (B) Cell viability of HSA-protamine conjugate as determined by MTT assay using arterial (pulmonary) smooth muscle cells $(n=8)$. Data represent mean \pm SD. ${ }^{* * *} p<0.001$. [Color figure can be viewed in the online issue, which is available at wileyonlinelibrary.com.]

Thus, it was confirmed that the higher enzymatic activity of tPA in the heparin-triggered group was not only because of heparin stimulation but also the removal of the albumin camouflage.

\section{DISCUSSION}

Use of HSA as a delivery tool has made seminal contribution in the field of controlled drug delivery. Albumin microspheres, for instance, have shown potential to deliver both small and large therapeutic molecules. ${ }^{7}$ Because of its natural abundance in blood, albumin-mediated delivery system can be ideal in designing a triggered release system for therapeutic macromolecules where the activity of the therapeutic cargo should be protected in blood. The key challenge in developing such delivery systems is masking the activity of the drug in the systemic circulation in a reversible manner so that pharmacologic action can be triggered at the disease site. As, unlike intracellular environment, blood environment lacks environmental triggers, such as $\mathrm{pH}$ or
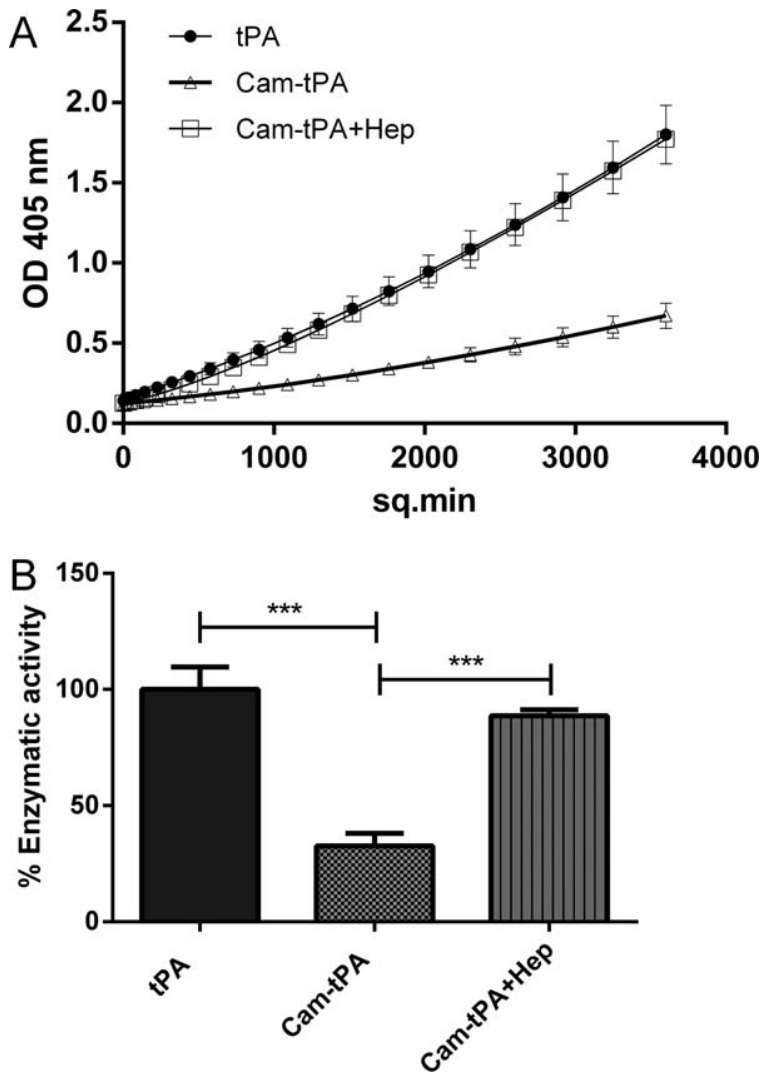

FIGURE 6. HSA-protamine conjugate in suppression and regeneration of activity of thrombolytic agent, tPA. (A) Chromogenic assay determining enzymatic action of tPA using plasmin-specific substrate S-2251. Experimental condition: HSA-protamine $0.2 \mu M$, S-2251 1.2 $\mathrm{m} M$, plasminogen $0.24 \mu M$, tPA $0.5 \mu \mathrm{g} / \mathrm{mL}$, and heparin $0.4 \mu / \mathrm{mL}$. (B) Enzymatic activity of tPA upon complex formation with HSA-protamine and subsequent regeneration with heparin. The activity was calculated from the slope of the enzymatic assay. Data represent mean $\pm \operatorname{SD}(\mathrm{n}-3) .{ }^{* *} p<0.001$.

reducing environment, regeneration of activity is often difficult. A classic example of protecting a protein in blood includes PEGylation of proteins to increase circulation halflife. However, covalent attachment of PEG on the protein surface, ironically, often results in diminished drug activity because of steric hindrance created by PEG molecules that prevent the target molecule to interact and produce pharmacological action, as was the case with tPA. ${ }^{22}$ Our proposed HSA-protamine conjugate is designed to address the above mentioned limitations in which the bulky albumin moiety will serve as a "camouflage" for the drug in the systemic circulation, whereas the positively charged protamine will facilitate formation of an electrostatic complex with the drug. Depending on the therapeutic application, a triggering agent can be chosen to disrupt the construct and liberate free drug at the site of action.

Preparing a one-to-one conjugate between two different proteins is desirable to avoid potential heterogeneity that might result from numerous reactive functional groups present on their surface. Considering the presence of the single, relatively reactive secondary amine group on the $\mathrm{N}$-terminus (proline) of protamine, and the free thiol group 
$\mathrm{BF}$

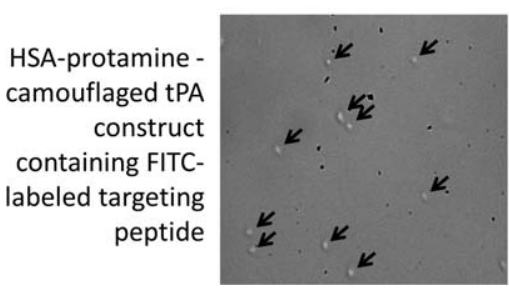

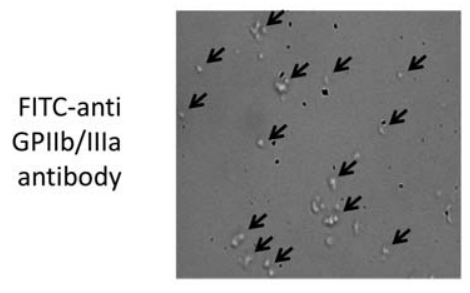

FITC
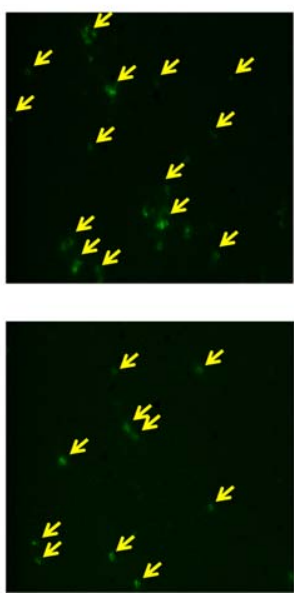

FIGURE 7. Binding of surface decorated HSA-protamine conjugate with activated platelets observed under fluorescence microscope (900× magnification). The bright field images indicate the adsorbed platelets while the FITC images staining with peptide-linked HSAprotamine conjugate. [Color figure can be viewed in the online issue, which is available at wileyonlinelibrary.com.]

on Cys-34 of HSA, reaction scheme was designed to link these two so that any heterogeneity can be avoided. However, a critical challenge was activation of the $\mathrm{N}$-terminal proline of protamine to make it thiol-reactive. Upon stabilization of protamine solution with high concentration of $\mathrm{NaCl}$ and PEG-600, a significant amount (63\%) of protamine was linked to SPDP that eventually demonstrated a spontaneous reaction with the Cys-34 of HSA. The chromatographic method employed to purify protamine from unreacted SPDP could not distinguish between unreacted and reacted protamine. However, it is important to note that, following conjugation with HSA, only the HSA-protamine conjugate was collected leaving the activated and nonactivated protamine that was eluted later [third fraction in the chromatogram; Fig. 3(C)]. The second fraction was the desired HSA-protamine conjugate, as evident by the electrospray mass spectrometry. The selection was initially based on partial charge screening because of the presence of albumin that would allow the conjugate to elute relatively earlier than native protamine, and also from the observation that upon increasing concentration of albumin, the second fraction reaches a higher peak with consequent reduction of the later fraction, which is assumed to be the unreacted protamine. Moreover, the collected fraction was finally confirmed as HSA-protamine conjugate by determining the molecular weight that was found to be consistent with the sum of the weights of HSA and protamine.

The hemolysis and cell viability study further suggest that the possible deleterious effect of cationic protamine is diminished by the presence of albumin. We argue that the immunogenicity associated with protamine use would also be attenuated upon conjugation with albumin. This argument is supported by earlier observation that conjugation of PEG molecules to protamine attenuates complement activation and subsequent immunogenic response ${ }^{19}$ because of the shielding effects created by PEG. As albumin, being several-folds larger than protamine, is conjugated to $\mathrm{N}$-terminus of protamine, a similar nonimmunogenic profile is expected. It should also be noted that albumin is the most abundant protein in human plasma that, along with its size, makes it an excellent choice as a "camouflaging moiety". Moreover, HSA offers a high degree of flexibility for surface modification that allows introducing homing moieties to direct the construct towards any desired site of action.

The proof-of-concept study using tPA showed convincing outcome in camouflaging tPA's enzymatic action upon complex formation with HSA-protamine conjugate, followed by regeneration of activity using therapeutic steady state heparin concentration. Therefore, the lytic action of tPA will be impeded in the systemic circulation but will be potentiated at the clot site, and hence will attenuate bleeding complication that can occur with conventional tPA therapy. The construct was designed such that tPA's action can be triggered using a triggering agent that is already included in the therapeutic modality of thrombosis management. Therefore, unlike other triggered-release systems, any external trigger, for example ultrasound, is not required. It should be noted that in the plasma condition, activity of the camouflaged tPA
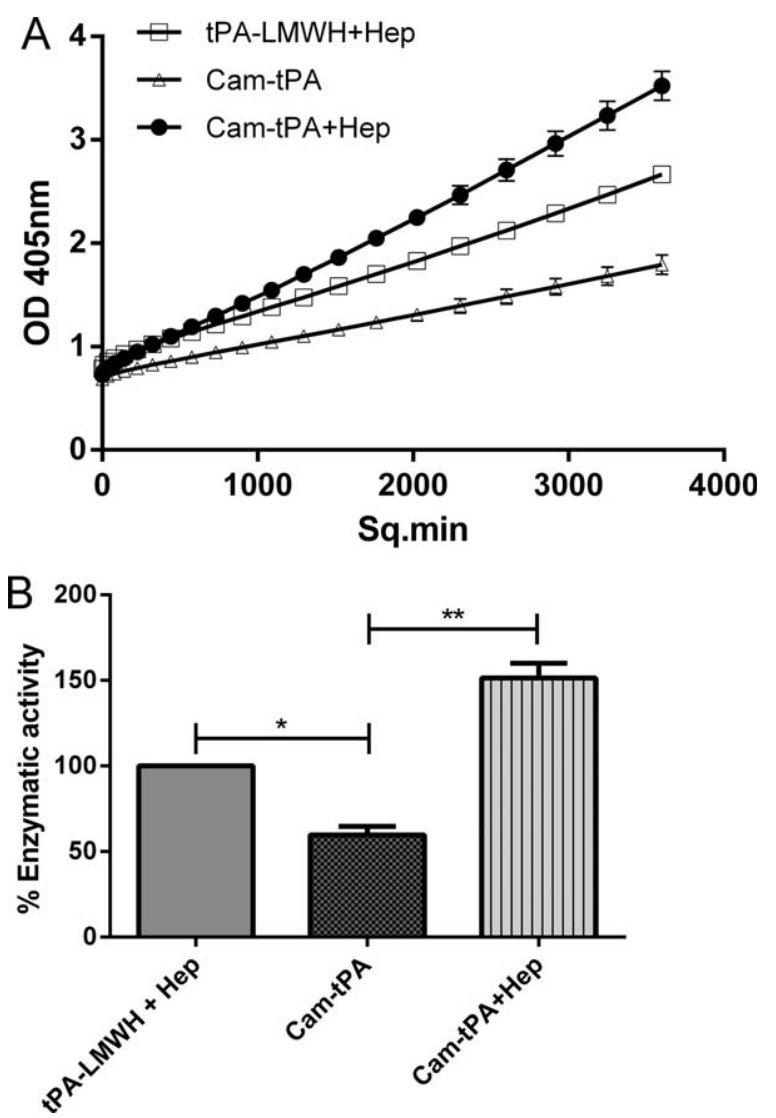

FIGURE 8. Stability of HSA-protamine mediated camouflaged tPA in human plasma. (A) Amidolytic activity of tPA determined after incubation in human plasma. (B) Enzymatic activity of tPA upon camouflaging action of HSA-protamine and triggered release by heparin. Data represent mean $\pm \mathrm{SD}(n=3)$. ${ }^{*} p<0.05,{ }^{*} p<0.01$. 
after regeneration in human plasma was even higher than that of native tPA. Based on the concept of steric stabilization, the shielding effect created by the HSA moiety can potentially attenuate PAI-1 mediated inactivation of tPA in plasma, while no such protection is available for the native tPA. This observation is in accordance with the hypothesis that, using HSA-protamine, the macromolecules will be protected not only from producing systemic action, but also from circulating inactivating molecules which eventually could offer a larger amount of active drug in the site of action, thereby minimizing the dose.

Along with thrombolytic agents, a potential application for this construct could be the delivery of anticancer drug for which minimization of systemic exposure is warranted. Moreover, the utility of albumin carrier to solid tumor through endothelial binding with GP60 is well documented. ${ }^{3-5}$ In this case, regeneration of activity can be achieved by reduction of disulfide bond between HSA and protamine at the intracellular compartment, offering active chemotherapeutic agent at the cancerous site. Similar approach can also be applied to intracellular delivery of genetic materials by protecting them in the systemic circulation, making this system a potentially widely applicable, biocompatible carrier of therapeutic macromolecules.

\section{CONCLUSION}

In this study, we have proposed a novel albumin-based delivery system for therapeutic macromolecules. A one-toone conjugate between HSA and protamine was synthesized and characterized for physical properties and biocompatibility. A proof-of-principle study using thrombolytic protein, tPA, as a model drug demonstrated that HSA-protamine conjugate can form a stable electrostatic complex with functionalized tPA and significantly mask its enzymatic activity. A suitable triggering agent, in this case heparin, disrupts the construct and regenerates tPA's activity. Hence, the proposed HSA-protamine conjugate holds the potential towards a newer triggered release approach for therapeutic proteins.

\section{ACKNOWLEDGMENT}

The authors would like to thank Mr. George Littlejohn at the Coffee Memorial Blood Center (Amarillo, TX) for providing us with human plasma sample. The authors are also thankful to Mr. Jim Windak at University of Michigan for the electrospray mass spectrometry work. This work was supported in part by the internal fund of TTUHSC and in part by Health Professions Division Research Grant from Nova Southeastern University.

\section{REFERENCES}

1. Fanali G, di Masi A, Trezza V, Marino M, Fasano M, Ascenzi P. Human serum albumin: From bench to bedside. Mol Aspects Med 2012;33:209-290.

2. Morales J. Defining the role of insulin detemir in Basal insulin therapy. Drugs 2007;67:2557-2584.

3. Schnitzer JE. gp60 is an albumin-binding glycoprotein expressed by continuous endothelium involved in albumin transcytosis. Am J Physiol 1992;262:H246-H254.
4. Tiruppathi C, Song W, Bergenfeldt M, Sass P, Malik AB. Gp60 activation mediates albumin transcytosis in endothelial cells by tyrosine kinase-dependent pathway. J Biol Chem 1997;272:2596825975.

5. Wang Z, Tiruppathi C, Minshall RD, Malik AB. Size and dynamics of caveolae studied using nanoparticles in living endothelial cells. ACS Nano 2009;3:4110-4116.

6. Guo $\mathrm{Q}$, Wang $\mathrm{H}$, Zhao $\mathrm{Y}$, Wang $\mathrm{H}$, Zeng $\mathrm{F}$, Hua $\mathrm{H}$, Xuc $\mathrm{Q}$, Huang $\mathrm{Y}$. Cell-penetrating albumin conjugates for enhanced doxorubicin delivery. Polym Chem 2013; DOI:10.1039/C3PY00742A.

7. Elsadek B, Kratz F. Impact of albumin on drug delivery-New applications on the horizon. J Control Release 2012;157:4-28.

8. Graeser R, Esser N, Unger H, Fichtner I, Zhu A, Unger C, Kratz F. INNO-206, the (6-maleimidocaproyl hydrazone derivative of doxorubicin), shows superior antitumor efficacy compared to doxorubicin in different tumor xenograft models and in an orthotopic pancreas carcinoma model. Invest New Drugs 2010;28:14-19.

9. Kratz F, Warnecke A, Scheuermann K, Stockmar C, Schwab J, Lazar P, Drückes P, Esser N, Drevs J, Rognan D, Bissantz C, Hinderling C, Folkers G, Fichtner I, Unger C. Probing the cysteine34 position of endogenous serum albumin with thiol-binding doxorubicin derivatives. Improved efficacy of an acid-sensitive doxorubicin derivative with specific albumin-binding properties compared to that of the parent compound. J Med Chem 2002;45: 5523-33.

10. Absar S, Choi S, Yang VC, Kwon YM. Heparin-triggered release of camouflaged tissue plasminogen activator for targeted thrombolysis. J Control Release 2012;157:46-54.

11. Absar S, Choi S, Ahsan F, Cobos E, Yang VC, Kwon YM. Preparation and characterization of anionic oligopeptide-modified tissue plasminogen activator for triggered delivery: An approach for localized thrombolysis. Thromb Res 2012;131:e91-e99.

12. Leach JK, O'Rear EA, Patterson E, Miao Y, Johnson AE. Accelerated thrombolysis in a rabbit model of carotid artery thrombosis with liposome-encapsulated and microencapsulated streptokinase. Thromb Haemost 2003;90:64-70.

13. Ware S, Donahue JP, Hawiger J, Anderson WF. Structure of the fibrinogen gamma-chain integrin binding and factor XIIla crosslinking sites obtained through carrier protein driven crystallization. Protein Sci 1999;8:2663-2671.

14. Patel B, Gupta V, Ahsan F. PEG-PLGA based large porous particles for pulmonary delivery of a highly soluble drug, low molecular weight heparin. J Control Release 2012;162:310-320.

15. Liu J, Guo S, Li Z, Liu L, Gu J. Synthesis and characterization of stearyl protamine and investigation of their complexes with DNA for gene delivery. Colloids Surf B Biointerfaces 2009;73: 36-41.

16. Naik SS, Liang JF, Park YJ, Lee WK, Yang VC. Application of "ATTEMPTS" for drug delivery. J Control Release 2005;101:35-45.

17. Absar S, Nahar K, Kwon YM, Ahsan F. Thrombus-targeted nanocarrier attenuates bleeding complications associated with conventional thrombolytic therapy. Pharm Res 2013;30:1663-1676.

18. Gupta AS, Huang G, Lestini BJ, Sagnella S, Kottke-Marchant K, Marchant RE. RGD-modified liposomes targeted to activated platelets as a potential vascular drug delivery system. Thromb Haemost 2005;93:106-114.

19. Chang LC, Lee HF, Chung MJ, Yang VC. PEG-modified protamine with improved pharmacological/pharmaceutical properties as a potential protamine substitute: Synthesis and in vitro evaluation. Bioconjug Chem 2005;16:147-155.

20. Meng F, Manjula BN, Smith PK, Acharya SA. PEGylation of human serum albumin: Reaction of PEG-phenyl-isothiocyanate with protein. Bioconjug Chem 2008;19:1352-1360.

21. Liang JF, Li Y, Yang VC. The potential mechanism for the effect of heparin on tissue plasminogen activator-mediated plasminogen activation. Thromb Res 2000;97:349-358.

22. Berger H Jr, Pizzo SV. Preparation of polyethylene glycol-tissue plasminogen activator adducts that retain functional activity: Characteristics and behavior in three animal species. Blood 1988; 71:1641-1647. 